\title{
ANÁLISIS DE LA INTRODUCCIÓN DE LA TEORÍA DE ENLACES Y BANDAS
}

\author{
SOLBES, J. y VILCHES, A. \\ Seminario de Física y Química. Servei de Formació Permanent de la Universitat de València.
}

\begin{abstract}
SUMMARY
This paper analyses different approaches to the introduction of students to basic concepts of the chemical bond and band theories, as well as common misconceptions related to each concept.
\end{abstract}

\section{INTRODUCCIÓN}

La enseñanza de las ciencias proporciona una imagen incorrecta de la ciencia marcada por el empirismo (Ausubel 1978, Gil 1983, Hodson 1985 y 1988, Millar y Driver 1987). Pero como hemos mostrado en trabajos anteriores (Gil, Senent, Solbes 1989), hay otra causa de dicha imagen incorrecta : una visión lineal y acumulativa de la ciencia que ignora la importancia de los paradigmas en la construcción del nuevo conocimiento hasta que dificultades insuperables producen una revolución teórica y el surgimiento de un nuevo paradigma. Esta imagen acumulativa se pone de manifiesto en el hecho mostrado repetidamente de que la física clásica se introduce sin tener en cuenta los preconceptos de los alumnos o, en dos palabras, las ideas que la física clásica desplazó (Posner et al. 1982, McDermott 1984, Gil y Carrascosa 1985 y Hashew 1986).

En varios trabajos anteriores hemos mostrado, mediante el análisis de textos y cuestionarios para profesores, que la física moderna se yuxtapone o incluso se mezcla con las concepciones clásicas sin mostrar la existencia de una neta ruptura entre ambas (Gil, Senent y Solbes 1986). Este tratamiento didáctico incorrecto va acompañado de graves errores en torno a conceptos clave: relaciones masa/energía (Gil; Senent y Solbes 1988), dualidad, indeterminación, etc. (Solbes 1986).

Asimismo al abordar los dominios en que se aplican las ideas cuánticas, por ejemplo la estructura atómica, encontramos también una yuxtaposición y/o mezcla de los modelos atómicos clásicos (Rutherford) semicuánticos, (Bohr, Sommerfeld) y cuánticos, y graves errores con- ceptuales, por ejemplo considerar el orbital atómico (o Ios niveles energéticos correspondientes) como zonas del espacio que existen independientemente de los electrones y que pueden ser ocupadas o no por éstos (Solbes et al. 1987 a y b).

¿Qué consecuencias tiene esto en los alumnos? Hemos encontrado (Gil, Senent y Solbes 1986 y 1989) que, en primer lugar, perjudica la correcta comprensión no sólo de la física moderna, sino también de la física clásica, al no tener claros los límites de validez de esta última y las diferencias entre ambos paradigmas, y proporciona una imagen deformada de cómo se desarrolla la ciencia y, por tanto, de la propia metodología científica. En segundo lugar, los alumnos incurren en la totalidad de los errores detectados en los textos, dado que en el dominio de Ia física moderna, los errores no son debidos tanto a las experiencias cotidianas previas, cuanto a la experiencia escolar anterior (y/o los medios de comunicación, etc.). Por otra parte, la ausencia de tratamientos diđácticos clarificadores, que muestren cómo la fúsica moderna entra en contradicción con la clásica y, por tanto, con la estructura conceptual del alumno, imposibilita el cambio conceptual de éste, y produce la aparición de nuevos errores.

Así, entre los alumnos de secundaria hemos encontrado, por ejemplo, transformaciones de masa en energía, limitar la dualidad onda-corpúsculo de toda la materia sólo a la Iuz, asociar la onda al movimiento del cuerpo, afirmar que el electrón no se puede localizar con precisión, etc. Es muy significativa la persistencia de dichos 
errores en alumnos de $2^{\circ}$ de físicas que consideran que: el electrón es un corpúsculo, la indeterminación impide medir con precisión, el orbital es una región espacial independiente de los electrones que puede ser ocupado o no por éstos (Solbes, Bernabeu et aI. 1988).

Estas breves consideraciones nos llevan a plantearnos qué tratamiento didáctico se realizará en los textos de física y química de BUP y COU en cualquier otro dominio en el que se apliquen las ideas de fisica moderna, concretamente, en el enlace químico y en la teoría de bandas de los sólidos.

\section{HIPÓTESIS}

Lo visto en el apartado anterior nos permite suponer que en los textos:

1. Aparezca una mezcla de modelos-clásicos, cuánticos, etc.- para los distintintos tipos de enlace. La diferencia con respecto al átomo consiste en que en éste se yuxtaponían modelos extrapolando su dominio de validez (por ejemplo, al modelo de órbitas se le hacían explicar los números cuánticos correctos). Por el contrario, aquí lo que se impide con dicha mezcla es una visión unitaria delenlace, cuya necesidad ya ha sido puesta de manifiesto anteriormente (Ducasse et al. 1985 y Aguirre 1985) al no poner de manifiesto que :

a) La descripción cuántica es válida no sólo para el enlace covalente, sino también para el iónico, metálico, etc.

b) Los diversos tipos de enlace son debidos a una única interacción: la electromagnética.

2. No se muestre que la descripción clántica proporciona información no sólo sobre las distribuciones de probabilidad espacial de los electrones, también la proporciona sobre los estados energéticos (niveles o bandas en moléculas y sólidos). Esto da pie a una distinción radical entre modelo de enlaces y modelo de banđas obstaculizando así la visión unitaria de las sustancias en estado sólido. El origen de éste estriba en considerar los orbitales únicamente como regiones del espacio, ya puesta de manifiesto en los textos españoles para los orbitales atómicos (Solbes et al. 1987) y detectada en estudiantes universitarios españoles (Solbes, Bernabeu et al. 1988) y franceses (Dumon y Merlin 1988).

Más en concreto, y con ello pasamos a operativizar nuestra hipótesis, cabe esperar que dichos textos:

1. Impidan la visión unitaria del enlace químico utilizando los modelos precuánticos de la teoría electrónica de valencia para el iónico (la transferencia de electrones de Kossel -1916-) y para el covalente (el par de electrones compartidos de Lewis -1916-); el modelo cuántico del enlace de valencia e incluso de orbitales moleculares, también para el enlace covalente; y el modelo clásico del gas de electrones libres de Drude -1900 - y Lorenz 1909- para el metálico.
2. Presenten los modelos como la descripción correcta y real, ignorando que se trata de aproximaciones sencillas con sus correspondientes limitaciones -por ejemplo, no se muestra que el modelo de Lewis era incapaz de calcular energías o longitudes de enlace, de justificar la existencia de pares: Lewis llegó a afirmar que la ley de Coulomb no era válida a distancias atómicas para justificarlos (Oliva 1987).

3. En consecuencia estos modelos -por ejemplo, el de Lewis - se extrapolan fuera de su dominio de validez para abordar determinados problemas -como resonancia, geometría molecular-, para lo cual es necesario añadir hipótesis suplementarias, híbridos de resonancia, teoría de la repulsión de los pares electrónicos de valencia. Con ello resulta más complejo dicho modelo que el correcto, como sucede con las conocidas complicaciones del modelo de Ptolomeo (epiciclos, ecuantes) o de Bohr (órbitas de Sommerfeld) para mantenerlos pese a las anomalías registradas.

4. No dejen claro que la cohesión de los átomos es de origen electromagnético, y que no puede explicarse clásicamente; ia única forma de hacerlo correctamente, es mediante los conceptos cuánticos que dan cuenta no sólo del enlace covalente, sino del iónico, metálico, etc.

5. No permitan que el alumno visualice, en algunos casos, cuál es la distribución electrónica del átomo en el enlace, es decir, no presenta los orbitales moleculares, aunque sea simplemente a partir de la superposición de orbitales atómicos, sin necesidad de introducir el método de orbitales moleculares:

6. No muestren que en la cuántica están relacionadas la distribución de probabilidad espacial de los electrones y sus niveles energéticos.

7. En consecuencia, sólo se presentará la distribución espacial, sin proporcionar información (aunque sea remitiendo a cursos superiores) sobre los niveles energéticos de las moleculas o las bandas de los sólidos, correspondientes a dichas distribuciones espaciales.

8. Otra consecuencia de no mostrar esta relación es el error conceptual de interpretar las funciones de estado u orbitales (moleculares, híbridos, etc.) únicamente como regiones del espacio (en conexión con $\psi$ ) sin mostrar que también determinan los estados $\mathrm{y}$, consecuentemente, los niveles energéticos.

A su vez, respecto a los temas de estado sólido/electrónica, que aparecen únicamente en los textos de física de COU, cabe esperar que:

1. Sólo se presenta la distribución energética (modelo de bandas). Se olvida así que ambos modelos, enlaces y banđas, corresponden a una única descripción cuántica.

2. Consecuentemente, no pongan de manifiesto las relaciones con los temas de enlaces químicos, al no mostrar qué tipo de enlace -salvo quizás el metálico-corresponde a las estructuras de bandas de semiconductores y aislantes. 
3. Introduzcan expresiones que puedan inducir al error conceptual de atribuir una interpretación espacial a las bandas.

4. Por otra parte, la persistencia de modelos puede aparecer al utilizar el modelo clásico del «mar» de electrones; se mantiene para explicar la conductividad, cuando en realidad este modelo, al considerar los electrones e iones como partículas clásicas en colisión, predice resistividades elevadas (Tipler 1985).

5 . Finalmente, cabe esperar, que no se explique propiedades de las sustancias (eléctricas, ópticas, mecánicas, etc.) utilizando ambos modelos, evidenciando así una vez más, la escasa relación que existe en los libros de texto entre la ciencia y sus aplicaciones, puesta de manifiesto en anteriores trabajos (Solbes y Vilches 1989).

\section{DISEÑO EXPERIMENTAL}

Con objeto de contrastar nuestra hipótesis, hemos elaborado un cuestionario para el análisis de textos de física y química de $2^{\circ}$ y $3^{\circ}$ de BUP y de física y química de COU.

\section{CUESTIONARIO PARA EL ANÁLISIS DE TEXTOS}

AUTOR

TITULO

EDITORIAL

CIUDAD

\section{QUÍMICA}

1. Plantea una visión unitaria del enlace.

En caso negativo qué modelo utiliza para el enlace

iónico..........covalente..........metálico.........

2. Muestra las linitaciones de dichos modelos

3. Mantiene el modelo de Lewis para abordar los problemas de: resonancia, geometría de las moléculas, etc.

4. Explica el enlace, la cohesión entre fos átomos.

5. Representa los $O M$ (sin que sea necesario recurrir al método de $\mathrm{OM}$ ). ¿Qué representaciones gráficas realiza de los enfaces?

6. Muestra la relación entre la distribución espacial de los electrones y la descripción energética.

7. Introduce niveies energéticos moleculares.

8. Identifica los orbitales (moleculares, híbridos, etc.) con regiones del espacio.

9. Explica propiedades de las sustancias a partir de los enlaces.

FISICA

1. Muestra ta relación entre la distribución espacial de los electrones y la descripción energética.

2. Relaciona el modelo de enlaces y el de bandas.

3. Introduce expresiones que puedan inducir a una interpretación espacial de las bandas.

4. Mantiene el modelo del «mar de electrones», explicando la conductividad de los metales a partir de las colisiones entre electrones e iones.

5. Explica propiedades de los sólidos con ambos modelos.
Hemos realizado un ensayo de los cuestionarios independientemente de los dos autores del trabajo, con los mismos textos para analizar su validez; posteriormente, se ha reelaborado el cuestionario a la luz de los resultados obtenidos, llegándose al definitivo que adjuntamos a continuación (Tabla I).

Cada hipótesis operativa coincide con una cuestión. En Ia 1 y la 5 se añade un aspecto descriptivo, cualitativo y no cuantificable que comentaremos en el análisis de resultados; se trata, como se puede apreciar, de verificar si para cada uno de los f́tems se introduce o no el aspecto en cuestión.

Para poder cuantificar dichos resultados hemos considerado los porcentajes sobre el total de libros analizados que contestan negativamente a cada cuestión (salvo en los ítems 3 y 5 de química y los ítems 3 y 4 de física, donde la posición que confirma nuestra hipótesis es la afirmativa).

El análisis de los textos con el cuestionario definitivo lo han realizado los autores independientemente, para comprobar así la fiabilidad de los resultados.

\section{ANÁLISIS DE RESULTADOS}

EI cuestionario ha sido aplicado a 48 libros de texto (ver resultados en la tabla adjunta) y a efectos de su análisis los dividimos en 4 grupos: $2^{\circ} \mathrm{FQ}$ (17), $3^{\circ} \mathrm{FQ}$ (13), COU química (8) y COU física (10) (a este último grupo se le pasó la úitima parte del cuestionario). En la tabla hemos expresado los resultados en porcentajes pot grupos y totales para los tres primeros.

Ítem 1. De acuerdo con nuestra hipótesis, se observa que en los textos de $2^{\circ}$ de BUP un $88,24 \%$ de ellos no ofrecen

Tabla I

CUESTION $2^{2} \mathrm{FQ}(\%) \quad{ }^{3} \mathrm{FQ}(\%) \quad$ COU $\mathrm{Q}(\%) \quad$ Media $(\%)$ COU F(\%)

\begin{tabular}{cccccc}
\hline 1 & 88,24 & 100,00 & 81,50 & 89,91 & 87,50 \\
\hline 2 & 100,00 & 92,30 & 75,00 & 89,10 & 100,00 \\
\hline 3 & 57,14 & 75,00 & 42,86 & 58,33 & 83,33 \\
\hline 4 & 94,11 & 100,00 & 75,00 & 89,70 & 75,00 \\
\hline 5 & 64,70 & 46,75 & 50,00 & 53,82 & 100,00 \\
\hline 6 & 100,00 & 100,00 & 100,00 & 100,00 & \\
\hline 7 & 100,00 & 61,54 & 62,50 & 74,68 & \\
\hline 8 & 44,40 & 72,72 & 66,67 & 61,26 & \\
\hline 9 & 70,59 & 53,85 & 62,50 & 62,31 & \\
\hline
\end{tabular}


una visión unitaria del enlace químico, ya que la mayoría usan el modelo precuántico de la teoría electrónica de valencia para el iónico y el covalente, pero para el metálico utilizan el modelo clásico del gas de electrones. Muy pocos textos introducen en 2 o la visión cuántica, y lo hacen sólo para el enlace covalente $(17,5 \%)$. En los textos de $3^{\circ}$, el porcentaje de una visión no unitaria es del $100 \%$ ya que aquí la mayoría utilizan Lewis y la descripción cuántica para el enlace covalente $(85 \%$ con mayor 0 menor nivel de complejidad, desde nubes a orbitales moleculares, pasando por superposición de orbitales atómicos), pero, en el iónico y metálico, se prosigue con los modelos de Kossel y el del gas electrónico, respectivamente. Sólo un $15 \%$ de los textos de $3^{\circ}$ utilizan el modelo de bandas para el enlace metálico. En los libros đe quiuimica de $\mathrm{COU}$, se confirma también nuestra hipótesis, ya que el $81,5 \%$ de los libros no presentan una visión unitaria del enlace químico. La mayoría introducen Lewis y la descripción cuántica para el covalente. Sólo un $12,5 \%$ usa la descripción cuántica en el enlace iónico y un $37,5 \%$ en el caso del metálico. Son, por tanto, un porcentaje elevado los que siguen introduciendo modelo clásico del gas de electrones y el modelo de Kossel.

Ítem 2. En un porcentaje casi del $90 \%$ de los casos $(89,9 \%)$ los libros de los tres niveles coinciden en no mostrar las limitaciones del modelo, es decir, aquellos hechos que no explicaba o predecía (el modelo de Lewis no aporta información cuantitativa, -la energía o la longitud de enlace-, no justifica la existencia de pares, etc.).

Ítem 3. En el caso de $2^{\circ}$ de BUP en general no se trata de mantener el modelo de Lewis, dado que no introducen otro, sino de su utilización en situaciones más complejas como resonancia y geometría de las moléculas que requieren hipótesis suplementarias. En $3^{\circ}$ y COU, aunque en su totalidad introducen un modelo cuántico, casi un $60 \%$ sigue realizando una descripción de la mayor parte de las situaciones (resonancia, geometría, enlaces múltiples, polaridad, etc.) con estructuras de Lewis.

ítem 4. En general se confirma nuestra hipótesis en eI sentido de que los textos no explican la cohesión entre los átomos. Así, la mayor parte de los textos de $2^{\circ}$ utilizan Ia explicación empírica de la regla del octete, sin justificar por qué es más estable; no se da pues una explicación unitaria electrostática (tan sólo en el enlace iónico). Algunos textos presentan la curva de energía potencial como un resultado empírico y, por tanto, no explican el mínimo de dicha curva. En $3^{2}$ y COU se insiste en el octete y sólo se aplica al iónico (y a veces al metálico) la explicación electrostática. En el covalente apenas se utiliza la explicación energética, de acuerdo con nuestra hipótesis, y los pocos que lo hacen no la relacionan con la distribución de probabilidad, por lo que no explican el mínimo. Algunos indican la existencia de una densidad electrónica internuclear, pero pocos la utilizan para explicar la estabilidad. Ningún texto justifica cómo es posible el emparejamiento y la existencia de situaciones enlazantes, pese a haber intoducido el spin.

Item 5 . En la mayoría de textos de 20 (un $64,7 \%$ ) no se representan los orbitales moleculares (OM); en general dominan las representaciones sencillas: esferas macizas para el iónico y el covalente, representaciones de Lewis, algunas utilizando órbitas. La minoría introduce representaciones cuánticas: nubes electrónicas, superficies límite de orbitales atómicas (OA), cuya superposición origina las superficies límite de OM, y curvas de isodensidad electrónica. En 3ํㅡ y COU disminuye el número de textos que no representan OM (se acerca al 50\%). En estos niveles se siguen utilizando las esferas en el iónico, Lewis para el iónico y el covalente (en algunos casos con órbitas), que en el caso del iónico pueden llevar a pensar que el enlace es entre dos átomos. La práctica totalidad introduce representaciones cuánticas aunque sólo para el enlace covalente. La mayoría representa las superficies límite de los $\mathrm{OA}$ o de orbitales híbridos $(\mathrm{OH})$ y muestra su superposición. Algunos, aunque no introducen el método de OM, representan las superficies límite de los $\mathrm{OM}$ indicando que se originan por superposición de $O A$, lo que nos parece correcto porque permite visualizar la situación en las moléculas. En algunos textos aparecen situaciones paradójicas como, por ejemplo, denominar orbitales a las superficies límite pero no a las curvas de isodensidad; y algunos representan el desplazamiento de densidad de probabilidad electrónica mediante curvas de isodensidad, pero en el iónico siguen utilizando orbitas-transferencia.

Ítem 6. De acuerdo con nuestra hipótesis del modelo espacial de los enlaces, prácticamente ningún texto muestra las dos informaciones que proporcionan los orbitales: ia distribución de probabilidad y los estados energéticos. De hecho, ningún texto de los que introducen niveles moleculares relaciona ambos aspectos, entre otras cosas porque no mencionan la existencia de OM antienlazantes.

Ítem 7. Como cabía esperar ningún texto de $2^{\circ}$ muestra la existencia de niveles moleculares y de bandas en sólidos, y pocos en $3^{\circ}$ y $\mathrm{COU}$, ni siquiera sin describirlos, remitiendo a la física del mismo curso.

Ítem 8. En un porcentaje atto de libros $(61,26 \%)$ se confirma nuestra hipótesis. Se reproducen los errores detectados en el análisis del modelo cuántico; así se identifica el $\mathrm{OA}$, el $\mathrm{OH}$, y el $\mathrm{OM}$ con su representación espacial, asociados en realidad al cuadrado del orbital, en particular con la representación mediante superficies Iímite. Resulta interesante desde este punto de vista comprobar que no se identifican con otras representaciones, como las curvas de isodensidad que por rotación generan dichas superficies, lo que pone de manifiesto la identidad orbital-región del espacio.

Ítem 9. La mayor parte de los textos de los tres niveles no explican las propiedades de las sustancias a partir de los enlaces. La mayoría sólo las mencionan, justificando alguna de forma aislada.

En cuanto al cuestionario de Física:

Ítem 1. De acuerdo con nuestra hipótesis un $87,5 \%$ de los textos no muestran la relación entre la distribución espacial de los electrones y la descripción energética. Incluso algunos textos se limitan como en el tema del enlace a la información espacial y no introducen las 
bandas, dado que se limitan a explicar la semiconductividad con el modelo de enlaces. Sólo un texto muestra implícitamente dicha relación al mencionar que «existen orbitales que se extienden por todo el cristal y cuyas energías difieren poco entre sí formando bandas...»

Ítem 2. Ningún texto presenta una relación sistemática entre las diversas estructuras de bandas y los distintos enlaces, sólo queda manifiesta la relación entre enlace metálico y bandas de conductor; el covalente en algunos casos se relaciona con los semiconductores, sin mostrar la existencia de sólidos covalentes con estructura de bandas aislantes (p.e. el diamante).

Ítem 3. Algunos de los textos analizados no introducen Ias bandas, pero la mayoría de los que las introducen utilizan expresiones como uel desplazamiento de un electrón en la banda de valencia», "los electrones de dicha banda se moverán libremente», «ningún electrón podrá cruzar dicha banda y por consiguiente habrá conducción», etc., que pueden inducir dicho error, con Io que se confirma nuestra hipótesis.

Ítem 4. En algunos textos se da la resistencia como un hecho empírico, sin intentar explicar su origen, pero todos aquéllos que intentan explicarla señalan que «es debida a los choques de los electrones con los iones de la red».

Ítem 5. No se muestra la convergencia de ambos modelos para explicar las propiedades macroscópicas. La mayor parte sólo se limita a explicar propiedades eléctricas con las bandas, dejando de lado otras como las ópticas, etc.

\section{CONCLUSIONES}

Los resultados del cuestionario confirman nuestras hipótesis de que no se proporciona una visión unitaria de Ios diversos tipos de enlaces y que, además, los modelos de enlaces y bandas se presentan sin relación entre sí, impidiendo una visión unitaria de las sustancias en estado sólido.

Esto no supone que nos opongamos a la utilización de modelos en los niveles en que corresponda. Por ello en

\section{REFERENCIAS BIBLIOGRÁFICAS}

AGUIRRE, I. y GÓMEZ, R., 1985. El enlace químico. Un juego de simulación en un entomo de interacctón social, Enseñanza de las Ciencias, núm. extra, 48.

AUSUBEL, D.P., 1978. Psicología Educativa. Un punto de vista cognitivo. (Trillas: México). los materiales que se están elaborando para la enseñanza secundaria, se utiliza la teoría electrónica de valencia (Lewis y Kossel) en tanto se utilicen los modelos de Rutherford o Bohr para el enlace (15-17 años), pero poniendo de manifiesto que se trata de aproximaciones con sus correspondientes limitaciones, sin abordar aquellos fenómenos que exigen mayor número de hipótesis auxiliares -resonancia y geometría- y mostrando el caracter unitario del enlace, debido a la interacción electromagnética.

En los niveles superiores (17-19 años) se utiliza la descripción cuántica unitaria, que se inicia en el enlace covalente porque, a través de los orbitales moleculares deslocalizados (p.e. en el benceno) y de la covalencia polar, permite una comprensión cuántica de los enlaces: metálico, cuya densidad electrónica se extiende por todo el sólido e iónico - un gran desplazamiento de la densidad electrónica hacia el más electronegativo pero sin transferencia total-; al ser tan elevada dicha transferencia permite la utilización del modelo iónico en determinados cálculos, como por ejemplo en la energía reticular.

Por otra parte, en el enlace covalente no se distingue entre métodos del enlace de valencia y de orbitales moleculares porque ambos son aproximaciones que conducen a resultados similares. Por ello se introducen los OM como nuevos estados resultantes de la superposición de orbitales atómicos mostrando Ia doble información que nos proporcionan: espacial, las superficies límite y diagramas de contorno, y energética, el desdoblamiento de niveles, y remitiendo a la física para bandas, y se muestra cómo desde ambos puntos de vista se explica el enlace. No se presentan más orbitales moleculares porque los enlazantes tienen en bastantes casos características similares a la superposición de los orbitales atómicos originales (p.e. densidades de carga concentrados en los mismos lugares).

Asimismo se han elaborado materiales para el estudio de sólidos en niveles superiores (17-19 años). Su principal característica es mostrar las relaciones entre ambas descripciones-los diversos tipos de enlace y sus correspondientes estructuras de bandas- y explicar con ellos las diversas propiedades: eléctricas, ópticas, etc. Por otra parte los enlaces facilitan notablemente la comprensión de los tipos y mecanismos de conducción de los semiconductores.
DUCASSE, A., LANANNE, J. R., LANANNE, P. y RAYEZ, I.C., 1985 . Concepts modernes sur l'estructure électronique des molécules et la liason chimique, Bulletin de l'Union des Physiciens, núm. 678, pp. 129-219.

DUMON, A. y MERLIN, A., 1988. Difficulties with molecular orbitals, Education in Chemistry, 25, pp., 49-52. 
GIL, D., 1983. Tres paradigmas básicos en la enseñanza de las ciencias, Enseñanza de las Ciencias, 1, pp. 26-33.

GIL, D. y CARRASCOSA, J., 1985. Science learning as a conceptual and methodological change, European Journal of Science Education, 7, pp. 231-236.

GIL, D., SENENT, F. y SOLBES, J., 1986. Análisis crítico de la introducción de la Física Moderna, Revista de Enseñanza de la Física, 2, pp. 16-21.

GIL, D., SENENT, F. y SOLBES, J., 1988. E=mc I $_{2}$ la ecuación más famosa de la física: una incomprendida, Revista española de Ff́sica, 2 (2), pp. 53-55.

GIL, D., SENENT, F. y SOLBES, J., 1989. La Física Moderna en la Enseñanza Secundaria: una propuesta fundamentada y unos resultados, Revista española de Física, 3(1), pp. 53-58.

HASHEWH, M.Z., 1986. Towards an explanation of conceptual change, European Journal of Science Education, 8, pp. 229-249.

HODSON, D., 1985. Philosophy of science, science and science education, Studies in Science Education, 12, pp. 25-57.

McDERMOTT, L.C., 1984. Critical Review of Research in the domain of Mechanics, Research on Physics Education, (Editions du CRNS : París).

MILLAR, R. y DRIVER, R., 1987. Beyond Processes,Studies in Science Education, 14, pp. 33-62.

OLIVA, A. ,1987. Mecànica quàntica; base i futur de la química, pp. 87-113 de La revolució quàntica. (Fundació caixa de Pensions: Barcelona).

POSNER, G.S., STRIKE, K.A., HEWSON, P.W. Y HERTZOG, W.A., 1982. Accomodation of a scientific conception: towards a theory of conceptual change, Science Education, 66, pp. 211-227.

SOLBES, J. ,1986. La introducción de los conceptos básicos de Física Moderna. Tesis Doctoral, Universitat de València.

SOLBES, J., BERNABEU, J., NAVARRO, J. y VENTO, V., 1988. Dificultades en la enseñanza/aprendizaje de la Física Cuántica, Revista Española de Física, 2 (1), pp. 22-27.

SOLBES, J., CALATAYUD, M.L., CLIMENT, J.B. y NAVARRO, J., 1987. Errores conceptuales en los modelos atomicos cuánticos, Enseñanza de las ciencias, 5, pp. 189-196.

SOLBES, J., CALATAYUD, M.L., CLIMENT, J.B. y NAVARRO, J., 1987. El modelo cuántico del átomo, Servei de Formació Permanent Universitat de València.

SOLBES, J., VILCHES, A., 1989. Interacciones Ciencia/Ténica/Sociedad: un instrumento de cambio actitudinal, $E n$ señanza de las Ciencias, 7, pp. 14-19.

TIPLER, A., 1980. Física Moderna. (Reverte: Barcelona). 